\title{
Improvement of microcirculatory impairment in patients with predialysis chronic kidney disease after AST-120 administration
}

Takayasu Ohtake ${ }^{1 *}$, Yasuhiro Mochida ${ }^{1}$, Kunihiro Ishioka ${ }^{1}$, Hidekazu Moriya ${ }^{1}$, Sumi Hidaka ${ }^{1}$, Masahiro Hirata ${ }^{2}$, Hirokazu Yamada ${ }^{3}$, Hiromichi Kumagai ${ }^{4}$ and Shuzo Kobayashi ${ }^{1}$

\begin{abstract}
Background: Endothelial dysfunction starts at an earlier stage in patients with chronic kidney disease (CKD), and induces macro- and microcirculatory impairments. We evaluated whether uremic toxin-lowering therapy could improve endothelial function in patients with CKD.

Methods: We performed a prospective interventional trial with 30 predialysis non-diabetic CKD patients who were administered oral adsorbent AST-120 (6 g/day) for 12 months. Surrogate markers of endothelial function including flow-mediated dilation (FMD) and skin perfusion pressure (SPP) were serially evaluated along with serum indoxyl sulfate (IS) levels and renal function. Renal function was evaluated based on blood urea nitrogen, serum creatinine (sCr) levels, and estimated glomerular filtration ratio (eGFR).

Results: Monthly decline in renal function (slope of reciprocal sCr) after AST-120 administration did not change compared to that during the pre-treatment period. However, serum IS levels significantly decreased at 3 months after AST-120 administration $(p<0.01)$, and it was sustained during the period of AST-120 administration $(p<0.01)$. Although FMD did not improve, SPP was constantly elevated after AST-120 administration, and was significantly higher at 12 months compared with baseline value $(69.7 \pm 14.6$ vs. $78.8 \pm 18.9 \mathrm{mmHg}, p<0.05)$. A significant correlation between the change of IS and SPP from baseline values was shown at 6 months after AST-120 administration ( $r=-0.558, p=0.02)$.
\end{abstract}

Conclusion: Microcirculatory impairment was improved after AST-120 administration in patients with predialysis CKD. Trial registration: UMIN, UMIN000013577. Registered on March 31, 2014,

Keywords: AST-120, Chronic kidney disease, Microcirculation, Skin perfusion pressure

\footnotetext{
* Correspondence: ohtake@shonankamakura.or.jp

${ }^{1}$ Nephrology, Immunology, and Vascular Medicine, Kidney Disease and Transplant Center, Shonan Kamakura General Hospital, 1370-1 Okamoto, Kamakura 247-8533, Japan

Full list of author information is available at the end of the article
}

(c) The Author(s). 2020 Open Access This article is licensed under a Creative Commons Attribution 4.0 International License, which permits use, sharing, adaptation, distribution and reproduction in any medium or format, as long as you give appropriate credit to the original author(s) and the source, provide a link to the Creative Commons licence, and indicate if changes were made. The images or other third party material in this article are included in the article's Creative Commons licence, unless indicated otherwise in a credit line to the material. If material is not included in the article's Creative Commons licence and your intended use is not permitted by statutory regulation or exceeds the permitted use, you will need to obtain permission directly from the copyright holder. To view a copy of this licence, visit http://creativecommons.org/licenses/by/4.0/ The Creative Commons Public Domain Dedication waiver (http://creativecommons.org/publicdomain/zero/1.0/) applies to the data made available in this article, unless otherwise stated in a credit line to the data. 


\section{Introduction}

Chronic kidney disease (CKD) is one of the major diseases worldwide alongside hypertension and diabetes mellitus. The number of hemodialysis (HD) patients has been increasing, and cardiovascular diseases (CVD) are the major causes of death among these patients [1]. CKD is known as a major independent risk factor for CVD, and the prognosis of CKD patients is strongly affected by CVD [2]. Therefore, therapeutic strategy against CKD should be targeted at both inhibition of CKD progression and inhibition of cardiovascular complication.

CKD is characterized by the accumulation of several uremic toxins. Among them, indoxyl sulfate (IS) is a well-known uremic substance. A precursor of IS, indole, is produced via transformation from dietary tryptophan by bacterial flora in the intestine [3]. After being absorbed by the intestine, indole is transformed to IS in the liver. IS accumulates along with renal dysfunction and induces endothelial dysfunction via monocyte activation [4] and oxidative stress [5, 6]. IS accumulation is closely associated with the progression of atherosclerosis in CKD patients [7, 8].

Oral charcoal adsorbent AST-120 (Kremezin', Kureha Corporation, Tokyo, Japan) adsorbs uremic toxins in the gastrointestinal tract and prevents toxins being absorbed into the body. Indole is adsorbed by AST-120 in the intestinal tract thereby decreasing the serum IS concentration [9]. In consideration of the role of IS in the progression of atherosclerosis in CKD patients, AST-120 might improve the endothelial dysfunction in these patients. Endothelial dysfunction, in conjunction with vascular calcification, constitutes "vascular failure" in CKD patients [10]. We thus performed an interventional clinical trial to assess whether AST-120 might improve endothelial dysfunction in patients with CKD.

\section{Methods}

\section{Subjects and study design}

This study was designed to evaluate whether AST-120 has a beneficial effect on endothelial function in patients with non-diabetic CKD. The study protocol conformed to the Declaration of Helsinki, and was approved by the institutional ethics committees (no. TGE00388-024). This clinical trial was registered with an official clinical trial registration site (UMIN 000013577) before study initiation.

Eligible subjects were enrolled based on the following inclusion criteria: (a) progressive CKD (stage G3a to G5); (b) men or women aged 20-80 years, and (c) written informed consent. Progressive CKD was defined as change of reciprocal serum creatinine $(\mathrm{sCr})$ value of more than $0.01 \mathrm{~mL} / \mathrm{mg} / \mathrm{month}$. The exclusion criteria were (1) presence of diabetes mellitus, (2) use of contrast enhancement agents within the entry period, (3) use of nonsteroidal anti-inflammatory drugs, (4) previous history of myocardial infarction or stroke, (5) peripheral arterial disease (PAD) with stent implantation to lower limbs' arteries, (6) obstruction of the gastrointestinal tract, (7) planned major amputation of the lower limbs within the study period, and (8) pregnancy or expected pregnancy.

The study design is presented in Fig. 1. Eligible patients were pre-registered at -6 months after written informed consent was obtained. If their reciprocal $\mathrm{sCr}$ values matched the criteria of progressive CKD for a subsequent 3 months, the patients were formerly registered at -3 months. The 3 months' period from -3 to 0 month was set as the self-control period to validate the change in IS, renal function, flow-mediated dilation (FMD), and skin perfusion pressure (SPP) without AST120 intervention. AST-120 at a daily dose of $6 \mathrm{~g}(2 \mathrm{~g}$ three times per day) was started at 0 month and continued for 12 months. Serum IS, renal function, FMD, and SPP were evaluated at $-3,0,3,6$, and 12 months.

\section{Endpoints and data management}

Primary endpoint was change in IS, and secondary endpoint was changes of renal function, FMD, and SPP in this study. Considering the results of previous reports, which demonstrated a significant beneficial effect of AST-120 on pulse wave velocity [11] and showed a drop-out rate of $20-30 \%$ from the study, forty patients were planned to be enrolled to provide a significant difference, at a 0.05 significance level with $80 \%$ statistical power. Data management and data monitoring were performed in an independent research center (Mirai Iryo Research Center Inc., Tokyo, Japan). Statistical analysis was performed by independent statisticians.

\section{Clinical information and laboratory data}

Clinical information including age, sex, blood pressure, body mass index (BMI), underlying disease, and comorbidity was collected from medical records. Laboratory data including blood urea nitrogen (BUN), sCr, calcium (Ca), inorganic phosphate $(\mathrm{Pi})$, total cholesterol, high-density lipoprotein cholesterol (HDL-C), low-density lipoprotein cholesterol (LDL-C), triglyceride (TG), albumin, c-reactive protein (CRP), fibrinogen, and hemoglobin were examined in an in-hospital laboratory. Estimated glomerular filtration ratio (eGFR) was calculated using the Japanese formula [12] and categorized according to the CKD stage [13]. Fasting blood glucose and insulin were measured to calculate insulin resistance: homeostasis model assessment as an index of insulin resistance (HOMA-IR) (fasting blood insulin $(\mu \mathrm{U} / \mathrm{mL}) \times$ fasting blood glucose $(\mathrm{mg} / \mathrm{dL}) /$ 405). HOMA-IR $2.5<$ is thought to indicate insulin resistance. Serum IS levels were measured using a previously reported high performance liquid chromatography method [14]. We measured total IS (free IS and protein- 


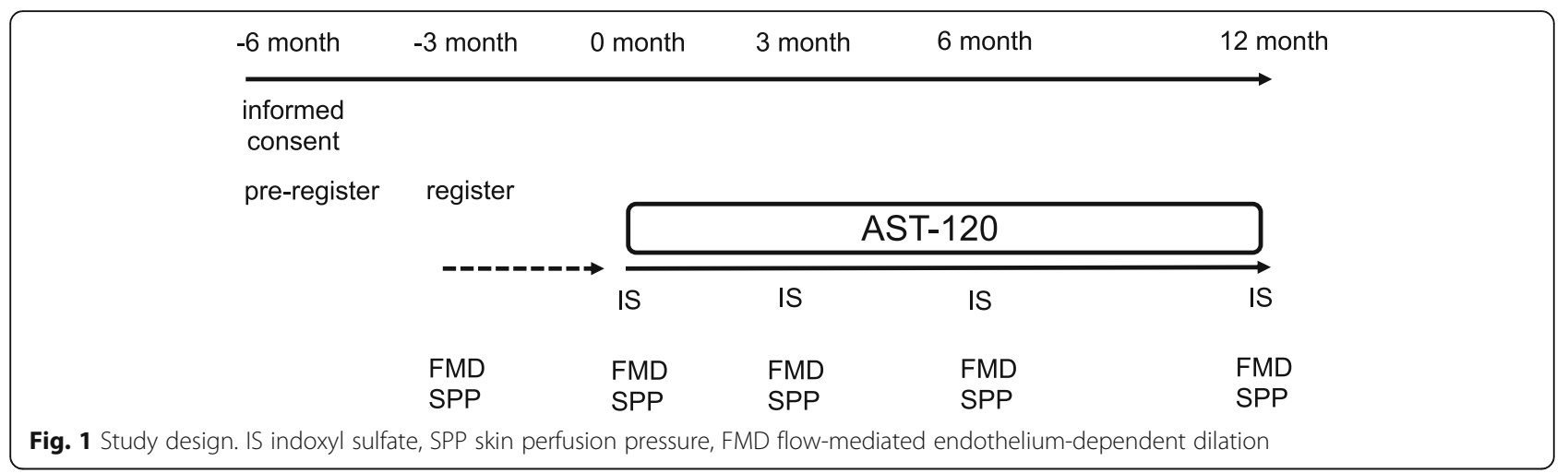

bound IS) and the data were expressed as $\mu \mathrm{M}$. Urinary protein and estimated dietary protein intake before and after AST-120 administration were also evaluated. Estimated protein intake was calculated using Maroni's formula [15].

\section{FMD and SPP}

FMD evaluates macrocirculatory status and SPP evaluates microcirculatory status just beneath the skin. FMD and SPP were measured using Endo-PAT2000 (Itamar Medical Japan, Tokyo, Japan) [16] and Laser Doppler (SensiLase PAD 3000, Kaneka Medix Corp., Osaka, Japan) [17], respectively, as previously reported. FMD was examined in the left upper arm of each patient. SPP was measured at a point between the first and second metatarsal bones in instep in each patient. Normal values of FMD and SPP are $\geqq 6 \%$ and $\geqq 70 \mathrm{mmHg}$, respectively.

\section{Statistical analysis}

All data are presented as the mean \pm standard deviation (SD) or median [IQR]. The two measured values of SPP in each patient at each time point were averaged and used for analysis. Skewed variables including CRP and TG with wide SD values underwent logarithmic transformation before statistical analysis. Difference in IS, renal function, FMD, SPP, and serum parameters were examined by one-way ANOVA and multiple comparison Scheffe test. Correlation between the changes of IS and SPP values was analyzed using Pearson's correlation analysis. SPSS statistics version 11.0 (SPSS Inc. Chicago, Illinois, USA) was used for data analysis and $p<0.05$ was considered significant.

\section{Results}

\section{Patients}

Thirty-four patients were registered between July 2014 and June 2017, and full analysis set (FAS) and per protocol set (PPS) comprised 30 and 24 patients, respectively. In the FAS group, twenty-three patients (76\%) were male, and the mean age was $74.0 \pm 7.6$ years old. The underlying kidney diseases were glomerulonephritis $(n=$ $25)$ and others or unknown $(n=5)$. Distribution of CKD stage was G3a $(n=4)$, G3b $(n=9)$, G4 $(n=14)$, and G5 $(n=3)$ (Table 1$)$. Mean $\mathrm{sCr}, 1 / \mathrm{sCr}$, and eGFR at 0 month were $2.03 \pm 0.85 \mathrm{mg} / \mathrm{dL}, 0.578 \pm 0.226 \mathrm{dL} / \mathrm{mg}$, and 29.3 $\pm 12.8 \mathrm{ml} / \mathrm{min} / 1.73 \mathrm{~m}^{2}$, respectively (Table 2 ). All patients had symptoms of chillness in their lower limbs.

\section{Changes of IS and renal function}

Mean serum IS levels significantly decreased at 3 months after AST-120 administration compared to the baseline values $(11.7 \pm 8.6 \mu \mathrm{M}$ to $6.9 \pm 5.0 \mu \mathrm{M}, p<0.001)$. A significant decrease in serum IS levels compared to baseline was sustained during AST-120 administration

Table 1 Baseline characteristics

\begin{tabular}{ll}
\hline Age (year) & $74.0 \pm 7.6$ \\
Male/Female, N & $23: 07$ \\
BMI (kg/m2) & $22.7 \pm 3.0$ \\
Blood pressure, mmHg & \\
Systolic & $129.5 \pm 14.6$ \\
Diastolic & $73.6 \pm 10.0$ \\
Underlying disease, N (\%) & \\
Glomerulonephritis & 25 \\
Others or unknown & 5 \\
CKD stage, N (\%) & \\
G3a & $4(13.3)$ \\
G3b & $9(30.0)$ \\
G4 & $14(46.7)$ \\
G5 & $3(10.0)$ \\
Comorbidity, N (\%) & \\
Hypertension & $25(83.3)$ \\
Dyslipidemia & $23(76.7)$ \\
Ischemic heart disease & $3(10.0)$ \\
Aortic aneurysm & $1(3.3)$ \\
\hline
\end{tabular}


Table 2 Change of renal function

\begin{tabular}{llllll}
\hline Month & -3 & 0 & 3 & 6 & 12 \\
\hline BUN (mg/dL) & $31.3 \pm 11.3$ & $31.1 \pm 12.1$ & $31.2 \pm 11.8$ & $34.2 \pm 15.4^{*}$ & $35.8 \pm 19.8^{*}$ \\
sCr (mg/dL) & $2.02 \pm 0.85$ & $2.03 \pm 0.85$ & $2.07 \pm 0.90$ & $2.21 \pm 1.00^{*}$ & $2.25 \pm 1.23^{*}$ \\
$1 / \mathrm{sCr}(\mathrm{dL} / \mathrm{mg})$ & $0.571 \pm 0.205$ & $0.578 \pm 0.226$ & $0.578 \pm 0.243$ & $0.550 \pm 0.251^{*}$ & $0.556 \pm 0.263$ \\
eGFR (mL/min/1.73m2) & $28.8 \pm 11.6$ & $29.3 \pm 12.8$ & $29.1 \pm 13.4$ & $27.6 \pm 13.7^{*}$ & $28.0 \pm 14.4$ \\
\hline
\end{tabular}

BUN blood urea nitrogen, $\mathrm{sCr}$ serum creatinine, eGFR estimated glomerular filtration ratio

${ }^{*} p<0.05$ vs. 0 month

for 12 months $(6$ month $8.0 \pm 6.4 \mu \mathrm{M}, 12$ months $8.8 \pm$ $5.9 \mu \mathrm{M})$ (Fig. 2).

Change in renal function is shown in Table 2. Levels of $\mathrm{sCr}$ gradually increased from baseline to 12 months. $\mathrm{sCr}$ values at 6 and 12 months were significantly higher than those at baseline. Monthly decline of $1 / \mathrm{sCr}$ (slope of $1 / \mathrm{sCr})(\mathrm{dL} / \mathrm{mg} /$ month) was not different between before and at 12 months after AST-120 administration. eGFR decreased at 6 months after AST-120 administration compared with that at baseline. However, eGFR at 12 months after AST-120 administration did not show statistically significant difference compared with that at baseline.

\section{Changes of atherosclerotic surrogate markers}

FMD did not show any significant change after AST-120 administration. However, SPP values were constantly elevated with time during AST-120 administration. SPP values at 12 months after AST-120 administration were significantly higher than the baseline values (baseline $69.7 \pm 14.6 \mathrm{mmHg}$ to 12 months $78.8 \pm 18.9 \mathrm{mmHg}, p<$ $0.05)$ (Table 3 and Fig. 3). There were 14 patients with SPP values below $70 \mathrm{mmHg}$ at baseline. In these patients, the rate of increase in SPP values from $<70$ to $\geqq 70 \mathrm{mmHg}$ was $57 \%(8 / 14)$ at 3 months, $50 \%(7 / 14)$ at 6 months, and $64 \%(9 / 14)$ at 12 months after AST-120 administration.

\section{Relationship between IS and SPP}

Absolute values of IS and SPP did not show a significant correlation before and at 3, 6, and 12 months after AST120 administration. However, change of IS and SPP from baseline levels showed a significant negative correlation at 6 months after AST-120 administration (Fig. 4). Patients with a more decrease in IS values showed a more increase in SPP values $(r=-0.558, p=0.02$, Fig. 4a). When these data were divided into CKD stage categories, a correlation between the decrease in IS values and increase in SPP values was found in patients with CKD stage G4 ( $r=-0.718, p=0.049$, Fig. $4 \mathrm{~b})$.

\section{Serum parameters}

Fibrinogen levels significantly increased at 12 months in contrast to the baseline value $(p<0.05)$, while CRP levels showed a borderline difference between baseline and 12 months $(p=0.05)$ (Table 4$)$. Serum albumin and

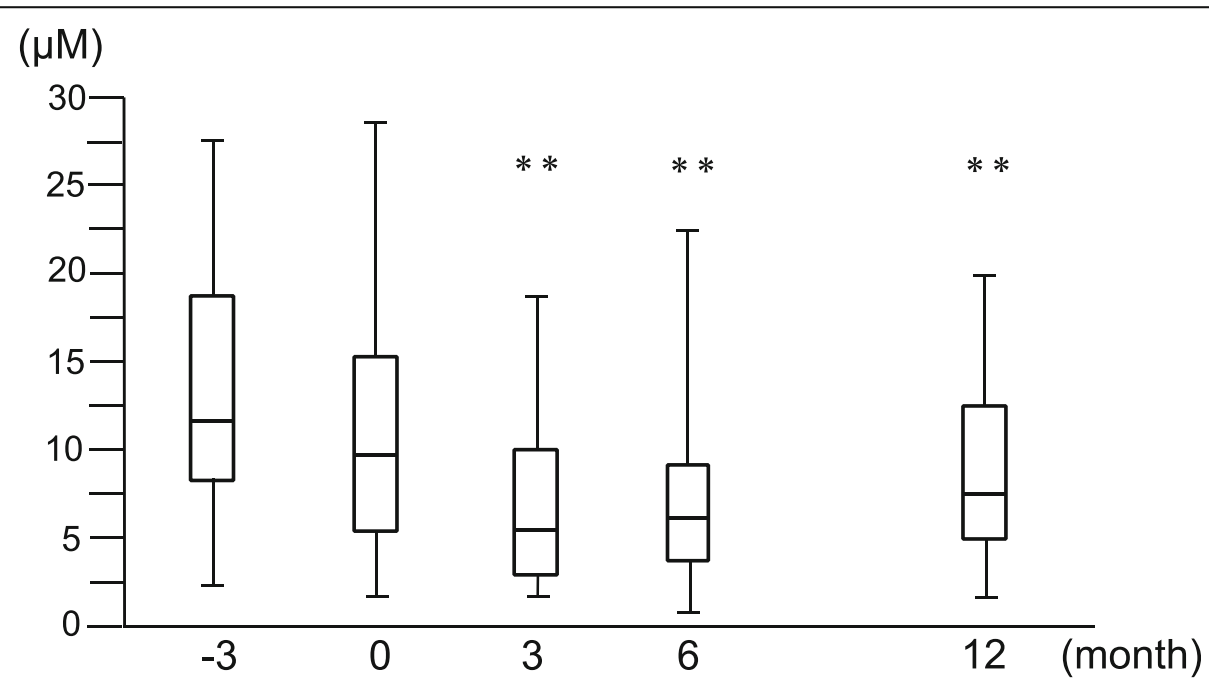

Fig. 2 Change of IS before and after AST-120 administration. Data are expressed as median [IQR]. ${ }^{* *} p<0.01$ vs. baseline value 
Table 3 Change of FMD and SPP

\begin{tabular}{llllll}
\hline Months & -3 & 0 & 3 & 6 & 12 \\
\hline FMD (\%) & $4.49 \pm 1.70$ & $4.03 \pm 2.80$ & $4.06 \pm 1.82$ & $3.85 \pm 1.85$ & $3.46 \pm 1.55$ \\
SPP $(\mathrm{mmHg})$ & $70.8 \pm 10.2$ & $69.7 \pm 14.6$ & $74.2 \pm 9.7$ & $75.0 \pm 10.6$ & $78.8 \pm 18.9^{*}$
\end{tabular}

FMD flow mediated dilation, $A C S$ arterial calciication score, SPP skin perfusion pressure

${ }^{*} p<0.05$ vs. 0 month

lipid profiles did not change during the study period. HOMA-IR also did not show any difference during the study period.

\section{Change of urinary protein and estimated daily protein intake}

There were 13 patients who had serial data of spot urine and 15 patients who had serial data of 24-h urine collection. Two patients did not have available serial urinary data. In 13 patients with serial spot urine data, urinary protein levels (gram/gram creatinine) did not change after the administration of AST-120 (0.66 \pm 0.94 at baseline, $0.68 \pm 0.61$ at 3 month, $0.67 \pm 0.85$ at 6 months, and $0.64 \pm 0.86$ at 12 months). In 15 patients with serial 24-h urine data, 24-h urinary protein levels (gram/day) also did not show a significant change after the administration of AST-120 (1.34 \pm 1.18 at baseline, $1.50 \pm 1.31$ at 3 months, $1.14 \pm 0.79$ at 6 months, and $1.63 \pm 1.31$ at 12 months, respectively).

In 15 patients with serial data of 24-h urinary urea nitrogen excretion, estimated dietary protein intake (gram/day) was not different before and after AST-120 administration ( $54.5 \pm 10.1$ at baseline, $53.4 \pm 9.2$ at 3 months, $51.4 \pm 11.2$ at 6 months, and $52.2 \pm 12.2$ at 12 months, respectively).

\section{Discussion}

In this interventional clinical trial, IS was significantly decreased after the initiation of AST-120 administration in CKD patients. The decline in renal function did not show a significant change as shown in a previous randomized controlled trial [18]. However, SPP values in the lower limbs constantly elevated and showed a significant improvement at 12 months after the initiation of AST-120 administration. Furthermore, more than half of the patients with SPP values below $70 \mathrm{mmHg}$ at baseline showed elevated SPP values to $70 \mathrm{mmHg}$ or more by 3 months or after longer periods of AST-120 administration. The change in IS values was significantly correlated with the change in SPP values at 6 months after AST120 administration. The beneficial effect of AST-120 on microcirculatory impairment might be related to a reduction of serum IS level, and renal functional improvement was not necessarily the determinant, as shown in an animal model [19].

Endothelial dysfunction could be divided into 2 categories, i.e., macrocirculatory and microcirculatory endothelial dysfunction. Endothelial dysfunction is associated with the progression of uremic milieu represented by IS accumulation, insulin resistance, and oxidative stress. Previous in vitro or animal studies provided that IS directly induced dose-dependent inhibition of proliferation, decrease in nitric oxide (NO) production, increase in reactive oxygen generation [6], and increase in intercellular adhesion molecule-1 (ICAM-1) and vascular cell adhesion molecule-1 (VCAM-1) mRNA [20] in human umbilical vein endothelial cells (HUVEC). These effects of IS on endothelial cells are thought to lead

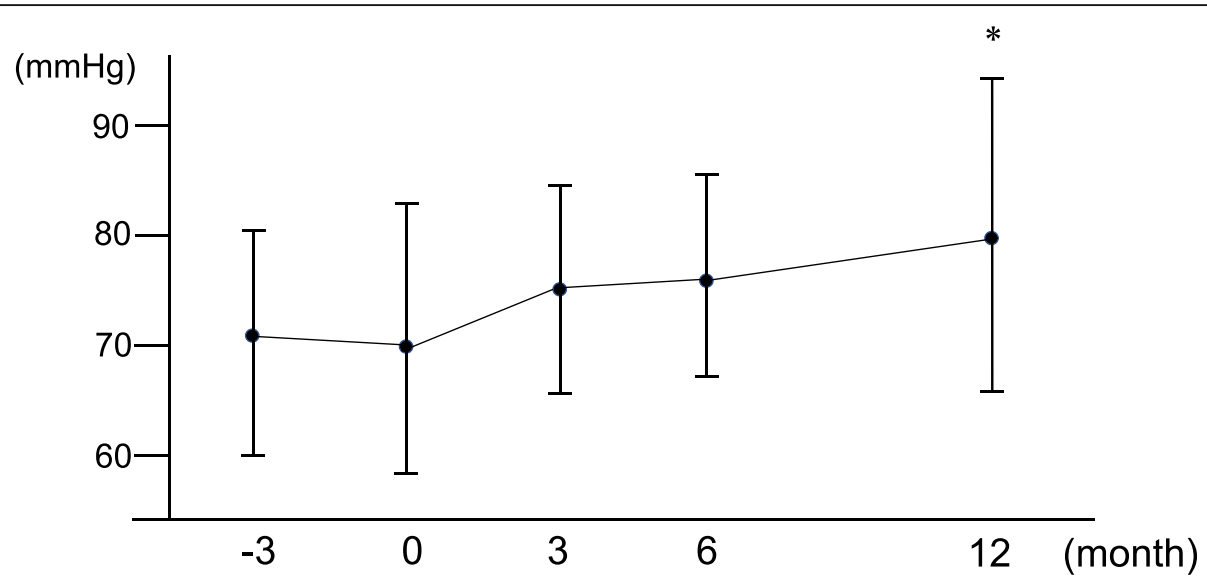

Fig. 3 Change of SPP before and after AST-120 administration. Data are expressed as mean \pm SD. * $p<0.05$ vs. baseline value 


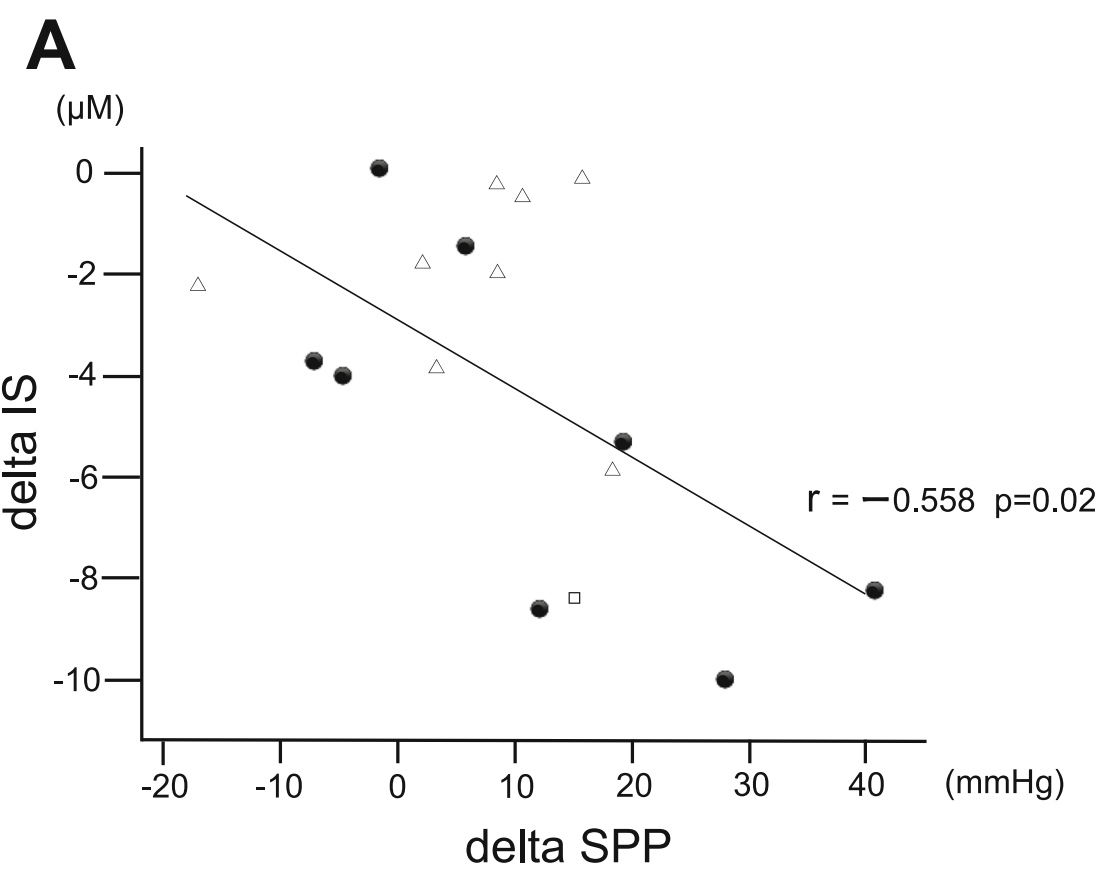

B

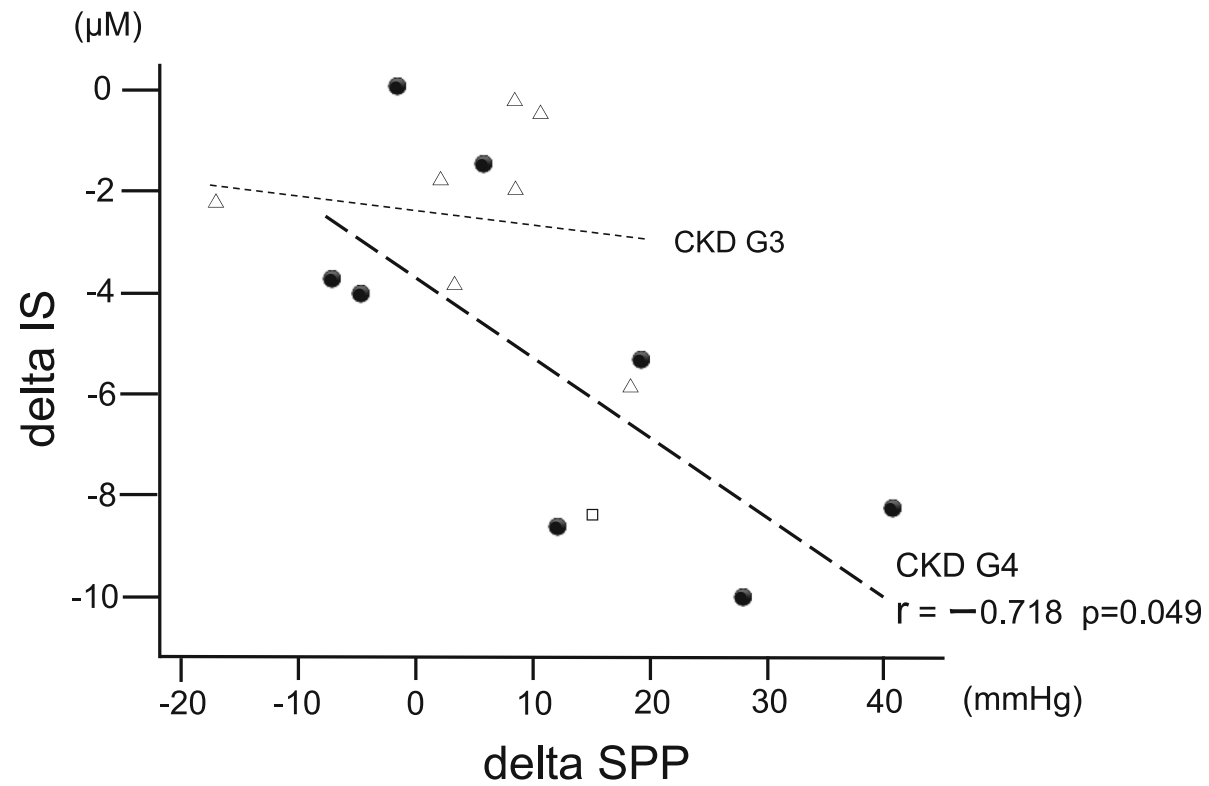

Fig. 4 Correlation between the changes of IS and SPP at 6 months after AST-120 administration. a Delta IS (changes from baseline values) showed a significant correlation with delta SPP (changes from baseline values) $(r=-0.558, p=0.02)$. b Patients with CKD stage G4 showed a strong correlation between IS change and SPP change at 6 months after AST-120 administration $(r=-0.718, p=0.049)$. $\Delta$ : CKD stage G3, •: CKD stage G4, $\square$ : CKD stage G5

endothelial dysfunction in CKD. We thus evaluated whether administration of AST-120 might improve endothelial dysfunction in predialysis CKD patients.

Microcirculatory endothelial function was improved after AST-120 administration (as indicated by the constant elevation of SPP), although improvement of macrocirculatory endothelial function could not be proven based on the FMD values. Only a few clinical reports wherein AST-120 administration improved the function of the vascular endothelium in CKD patients have been published $[6,21]$. Yu et al. reported improvement of FMD by 24 weeks of AST-120 administration in 
Table 4 Change of laboratory data

\begin{tabular}{llllll}
\hline Month & -3 & 0 & 3 & 6 & 12 \\
\hline Fibninogen (mg/dL) & $295.0 \pm 66.1$ & $296.8 \pm 71.3$ & $301.9 \pm 79.9$ & $304.2 \pm 75.4$ & $318.9 \pm 62.1^{*}$ \\
CRP (mg/dL) & $0.1 \pm 0.1$ & $0.1 \pm 0.3$ & $0.3 \pm 0.8$ & $0.3 \pm 0.9$ & $0.5 \pm 1.0$ \\
Albumin (g/dL) & $3.9 \pm 0.3$ & $3.9 \pm 0.3$ & $3.9 \pm 0.4$ & $4.0 \pm 0.3$ & $3.9 \pm 0.3$ \\
HOMA-IR & $7.5 \pm 11.8$ & $6.6 \pm 7.2$ & $7.7 \pm 10.5$ & $8.6 \pm 12.8$ & $8.8 \pm 15.0$ \\
T.cho (mg/dL) & $186.7 \pm 32.6$ & $187.0 \pm 29.3$ & $183.3 \pm 30.0$ & $186.1 \pm 32.0$ & $186.4 \pm 26.6$ \\
TG (mg/dL) & $194.3 \pm 133.5$ & $217.0 \pm 227.4$ & $175.9 \pm 96.9$ & $177.9 \pm 112.9$ & $208.1 \pm 196.8$ \\
HDL-C (mg/dL) & $48.5 \pm 11.5$ & $50.4 \pm 12.6$ & $50.3 \pm 12.2$ & $50.9 \pm 12.3$ & $52.3 \pm 12.9$ \\
LDL-C (mg/dL) & $99.0 \pm 30.6$ & $96.9 \pm 26.2$ & $97.9 \pm 27.9$ & $98.6 \pm 30.4$ & $97.6 \pm 27.4$ \\
Ca (mg/dL) & $9.0 \pm 0.4$ & $8.9 \pm 0.5$ & $8.9 \pm 0.4$ & $8.9 \pm 0.5$ & $8.8 \pm 0.5$ \\
Pi (mg/dL) & $3.3 \pm 0.6$ & $3.3 \pm 0.7$ & $3.4 \pm 0.7$ & $3.3 \pm 0.7$ & $3.6 \pm 0.9^{*}$ \\
CaxPi (mg2/dL2) & $29.8 \pm 5.2$ & $29.2 \pm 6.4$ & $30.4 \pm 6.2$ & $29.6 \pm 5.8$ & $31.7 \pm 7.5^{*}$ \\
\hline
\end{tabular}

CRP C-reactive protein, HOMA-IR homeostasis model assessment insulin resistance, T.cho total cholesterol, $T G$ triglyceride, $H D L-C$ high-density lipoprotein cholesterol, $L D L-C$ low-density lipoprotein cholesterol, $C a$ calcium, $P i$ inorganic phosphate ${ }^{*} p<0.05$ vs. 0 month

predialysis CKD patients [6], and Ryu et al. reported improvement of microvascular endothelial function by AST-120 administration in HD patients [21], respectively. The reason why the response of FMD and SPP differed in this study is not clear.

A beneficial effect of decreasing IS levels on the improvement of endothelial dysfunction was suggested (Fig. 4). Especially, our data suggested a beneficial association in patients with CKD stage G4. A mild association was also suggested in patients with CKD stage G3. However, it was not statistically significant. It might be due to the small number of patients with CKD stage G3 and G5. Therefore, a future clinical trial with more number in CKD stage G3 and G5 (early and late CKD stages) might be mandatory to clarify the association of IS and SPP in patients with these CKD stages.

Endothelial dysfunction emerges at an early stage of CKD along with IS accumulation, and insulin resistance (the upstream pathophysiological mechanism of atherosclerosis) is thought to be related to the endothelial dysfunction in CKD patients [22]. Therefore, we also evaluated whether insulin resistance as a role for endothelial dysfunction would improve after AST-120 administration. However, we could not demonstrate an improvement in the HOMA-IR by AST-120 administration in this study. Although we could not provide the clear mechanism underlying endothelial function improvement during AST-120 administration, this phenomenon might be related to other mechanisms than insulin resistance. A recent study also reported that IS suppresses endothelial progenitor cell (EPC)-mediated neovascularization in an animal model of hindlimb ischemia [23]. Microcirculatory impairment in CKD might be related to the disruption of vascular repair, which is mediated by EPC. Therefore, EPC potential in CKD patients and the effect of AST-120 administration also need to be evaluated in the future.
This study has limitations. First, fewer patients than that planned during study initiation were enrolled. Second, data on morphological change such as carotid intima-media thickness or left ventricular mass index, related to the change in endothelial function were not evaluated. Third, markers of oxidative stress were not evaluated in this study. And finally, this study evaluated surrogate markers of endothelial function, not hard endpoint, and there was no comparison with a control group of patients who were not prescribed AST-120. This study was a single arm study with a small sample size. Therefore, we evaluated data in -3 months and 0 months for data validation. A randomized controlled trial would be warranted to further evaluate the effect of IS-lowering therapy for endothelial function in CKD patients.

In conclusion, AST-120 administration significantly reduced IS concentration, and microcirculatory endothelial dysfunction was significantly improved after AST120 administration in patients with predialysis CKD. SPP values were significantly elevated by AST 120 administration, and more than half of the enrolled CKD patients showed elevation in the SPP values from $<70$ to $\geqq 70 \mathrm{mmHg}$ during the administration period. A correlation between the decrease in IS and improvement of SPP was also shown in this interventional study. Altogether, we conclude that IS-lowering therapy might be an effective therapeutic tool in predialysis CKD patients with microcirculatory impairment.

\section{Abbreviations}

BMI: Body mass index; BUN: Blood urea nitrogen; Ca: Calcium; CKD: Chronic kidney disease; CRP: C-reactive protein; CVD: Cardiovascular disease; eGFR: Estimated glomerular filtration ratio; EPC: Endothelial progenitor cell; FAS: Full analysis set; FMD: Flow-mediated dilation; HD: Hemodialysis; HDLC: High-density lipoprotein cholesterol; HOMA-IR: Homeostasis model assessment as an index of insulin resistance; HUVEC: Human umbilical vein endothelial cell; ICAM-1: Intercellular adhesion molecule-1; IQR: Interquartile 
range; IS: Indoxyl sulfate; NO: Nitric oxide; LDL-C: Low-density lipoprotein cholesterol; PAD: Peripheral arterial disease; Pi: Inorganic phosphate; PPS: Per protocol set; sCr: Serum creatinine; SD: Standard deviation; SPP: Skin perfusion pressure; TG: Triglyceride; VCAM-1: vascular cell adhesion molecule-1

\section{Acknowledgements}

We deeply thank Mrs. Tomoko Machida and Mr. Naoto Utada in Mirai Iryo Research Center, and clinical research coordinators in our hospital for their corporation in data assembly and data management.

\section{Authors' contributions}

Takayasu Ohtake: principal investigator, conception and design, provision of study material of patients, and manuscript writing. Yasuhiro Mochida: provision of study material of patients. Kunihiro Ishioka: provision of study material of patients. Hidekazu Moriya: provision of study material of patients. Sumi Hidaka: provision of study material of patients. Masahiro Hirata: data analyses as statistician. Hirokazu Yamada: data analyses as statistician. Hiromichi Kumagai: investigator of IS measurement. Shuzo Kobayashi: conception and design, data interpretation, and final approval of manuscript. All authors read and approved the final manuscript.

\section{Funding}

This study was funded by Mitsubishi Tanabe Pharma Corporation (Osaka, Japan).

\section{Availability of data and materials}

Please contact author for data requests. If it is an appropriate request, we will willingly provide our data.

\section{Ethics approval and consent to participate}

All procedures performed in this study involving human participants were in accordance with the ethical standards of our institutional ethical committee (IRB approval number TGE 00388-024) and with the 1964 Helsinki declaration and its later amendments.

\section{Consent for publication}

Written informed consent was obtained from all individual participants included in the study.

\section{Competing interests}

We declare that we have no competing interests.

\section{Author details}

${ }^{1}$ Nephrology, Immunology, and Vascular Medicine, Kidney Disease and Transplant Center, Shonan Kamakura General Hospital, 1370-1 Okamoto, Kamakura 247-8533, Japan. ${ }^{2}$ Center for Clinical and Translational Science, Shonan Kamakura General Hospital, Kamakura, Japan. ${ }^{3}$ Soiken Inc., Osaka, Japan. ${ }^{4}$ The Department of Clinical Nutrition, School of Food and Nutritional Sciences, University of Shizuoka, Shizuoka, Japan.

\section{Received: 15 November 2019 Accepted: 19 May 2020}

Published online: 29 June 2020

\section{References}

1. Nitta K, Masakane I, Hanafusa N, Taniguchi M, Hasegawa T, Nakai S, Goto S, Wada A, Hamano T, Hoshino J, Joki N, Abe M, Yamamoto K, Nakamoto H. The Japanese Society for Dialysis Therapy: An overview of Regular Dialysis Treatment in Japan (as of Dec. 31, 2017), edited by Nitta K et al. Tokyo: Japanese Society for dialysis Therapy; 2017.

2. Sarnak MJ, Levey AS, Schoolwerth AC, Coresh J, Culleton B, Hamm LL, McCullough PA, Kasiske BL, Kelepouris E, Klag MJ, Parfrey P, Pfeffer M, Raij L, Spinosa DJ, Wilson PW. Kidney Disease as a Risk Factor for Development of Cardiovascular Disease: a Statement from the American Heart Association Councils on Kidney in Cardiovascular Disease, High Blood Pressure Research, Clinical Cardiology, and Epidemiology and Prevention. Circulation. 2003;108: 2154-69.

3. Niwa T, Nomura T, Sugiyama S, Miyazaki T, Tsukushi S, Tsutsui S. The protein metabolite hypothesis, a model for the progression of renal failure: an oral adsorbent lowers indoxyl sulfate levels in undialyzed uremic patients. Kidney Int Suppl. 1997;62:S23-8.
4. Ito S, Higuchi Y, Yagi Y, Nishijima F, Yamato H, Ishii H, Osaka M, Yoshida M Reduction of indoxyl sulfate by AST-120attenuates monocyte inflammation related to chronic kidney disease. L Leukoc Biol. 2013;93:827-45.

5. Yisireyili M, Shimizu H, Saito S, Enomoto A, Nishijima F, Niwa T. Indoxyl sulfate promotes cardiac fibrosis with enhanced oxidative stress in hypertensive rats. Life Sci. 2013;92:1180-5.

6. Yu M, Kim YJ, Kang DH. Indoxyl sulfate-induced endothelial dysfunction in patients with chronic kidney disease via an induction of oxidative stress. Clin J Am Soc Nephrol. 2011;6:30-9.

7. Taki K, Nakamura S, Miglinas M, Enomoto A, Niwa T. Accumulation of indoxyl sulfate in OAT1/3-positive tubular cells in kidneys of patients with chronic renal failure. J Ren Nutr. 2006;16:199-203.

8. Niwa T. Indoxyl sulfate is a nephron-vascular toxin. J Ren Nutr. 2010;20(5 Suppl):S2-6

9. Schulman G, Agarwal R, Acharya M, Beri T, Blumenthal S, Kopyt N. A multicenter, randomized, double-blind, placebo controlled, dose-ranging study of AST-120 (Kremezin) in patients with moderate to severe CKD. Am J Kidney Dis. 2006;47:565-77.

10. Kobayashi S. cardiovascular events in chronic kidney disease (CKD) - an importance of vascular calcification and microcirculatory impairment. Renal Replacement Therapy. 2016;2:55.

11. Nakamura T, Kawagoe Y, Matsuda T, Ueda Y, Shimada N, Ebihara I, Koide H. Oral adsorbent AST-120 decreases carotid intima-media thickness and arterial stiffness in patients with chronic renal failure. Kidney Blood Press Res. 2004;27:121-6.

12. Matsuo S, Imai E, Horio M, Yasuda Y, Tomita K, Nitta K, Yamagata K, Tomino Y, Yokoyama H, Hishida A. Revised equations for estimated GFR from serum creatinine in Japan. Am J Kidney Dis. 2009;53:982-92.

13. The Japanese Society of Nephrology: Clinical Practice Guidebook for Diagnosis and Treatment for Chronic Kidney Disease 2012, Tokyo Igakusha, Tokyo, Japan.

14. de Loor H, Meijers BKI, Meyer TW, Bammens B, Verbeke K, Dehaen W, Evenepoel P. Sodium octanoate to reverse indoxyl sulfate and $p$-cresyl sulfate albumin binding in uremic and normal serum during sample preparation followed by fluorescence liquid chromatography. J Chromatogr. A. $2009 ; 1216: 4684-8$

15. Maroni BJ, Steinman TI, Mitch WE. A method for estimating nitrogen intake of patients with chronic renal failure. Kidney Int. 1985;27:58-65.

16. Ohtake T, Oka M, Ikee R, Mochida Y, Moriya H, Hidaka S, Kobayashi S. Impact of lower limbs' arterial calcification on the prevalence and severity of PAD in patients on hemodialysis. J Vasc Surg. 2011;53:676-83.

17. Celemajer DS, Sorensen K, Ryalls M, Robinson J, Thomas O, Leonard JV, Deanfield JE. Impaired endothelial function occurs in the systemic arteries of children with homozygous homocysteinemia but not in their heterozygous parents. J Am Coll Cardiol. 1993;22:854-8.

18. Akizawa T, Asano Y, Morita S, Wakita T, Onishi Y, Fukuhara S, Gejyo F, Matsuo S, Yorioka N, Kurokawa K. Effect of a carbonaceous oral adsorbent on the progression of CKD: a multicenter, randomized, controlled trial. Am J Kidney Dis. 2009;54:459-67.

19. Namikoshi T, Tomita N, Satoh M, Sakuta T, Kuwabara A, Kobayashi S, Higuch Y, Nishijima F, Kashihara N. Oral adsorbent AST-120 ameliorates endothelial dysfunction independent of renal function in rats with subtotal nephrectomy. Hypertens Res. 2009;32:194-200.

20. Inami $Y$, Hamada C, Seto T, Hotta Y, Aruga S, Inuma J, Azuma K, lo H, Kaneko K, Watada H, Tomino Y. Effect of AST-120 on endothelial dysfunction in adenine-induced uremic rats. Int J Nephrol. 2014;2014: 164125.

21. Ryu JH, Yu M, Lee S, Ryu DR, Kim SJ, Kang DH, Choi KB. AST-120 improves microvascular endothelial dysfunction in end-stage renal disease patients receiving hemodialysis. Yonsei Med J. 2016;57:942-9.

22. Kobayashi S, Maesato K, Moriya H, Ohtake T, Ikeda T. Insulin resistance in patients with chronic kidney disease. Am J Kidney Dis. 2005;45:275-80.

23. Hung SC, Kuo KL, Huang HL, Lin CC, Tsai TH, Wang CH, Chen JW, Lin SJ, Huang PH, Tamg DC. Indoxyl sulfate suppresses endothelial progenitor cellmediated neovascularization. Kidney Int. 2016;89:574-85.

\section{Publisher's Note}

Springer Nature remains neutral with regard to jurisdictional claims in published maps and institutional affiliations. 